\title{
SPECTRAL PICTURES \\ OF HYPONORMAL BILATERAL OPERATOR WEIGHTED SHIFTS
}

\author{
DOMINGO A. HERRERO
}

(Communicated by Palle E. T. Jorgensen)

\begin{abstract}
This note provides a complete description of the spectral picture of a hyponormal bilateral operator weighted shift of finite multiplicity. If the weights are $m \times m$ matrices, then the operator cannot by cyclic, unless it is a normal operator of a very special kind. An example shows that, nevertheless, there exists a cyclic, nonnormal, hyponormal bilateral operator weighted shift whose weights are operators acting on an infinite dimensional Hilbert space. (This answers a question of J. B. Conway.)
\end{abstract}

\section{INTRODUCTION}

Let $\mathscr{L}(\mathscr{H})$ denote the algebra of all (bounded linear) operators acting on a complex, separable Hilbert space $\mathscr{H}$, and let $\left\{A_{n}\right\}(-\infty<n<\infty)$ be a uniformly bounded two-sided sequence of operators. The bilateral operator weighted shift $B$ (with weight sequence $\left\{A_{n}\right\}$ ) is the operator on $\ell^{2}(\mathscr{\ell}):=$ $\ell^{2} \otimes \mathscr{H}$ defined by

$$
B\left(\ldots, x_{-1},\left[x_{0}\right], x_{1}, \ldots\right)=\left(\ldots, A_{-2} x_{-2},\left[A_{-1} x_{-1}\right], A_{0} x_{0}, \ldots\right),
$$

where $x=\left(\ldots, x_{-1},\left[x_{0}\right], x_{1}, \ldots\right) \in \ell^{2}(\mathscr{H}) ;\left[x_{0}\right]$ denotes the central $(0$ th $)$ term of the two-sided sequence.

The adjoint of $B$ is defined by a similar equality

$$
B^{*}\left(\ldots, x_{-1},\left[x_{0}\right], x_{1}, \ldots\right)=\left(\ldots, A_{-1}^{*} x_{0},\left[A_{0}^{*} x_{1}\right], A_{1}^{*} x_{2}, \ldots\right),
$$

and the self-commutator $\left[B^{*}, B\right]=B^{*} B-B B^{*}$ of $B$ is the "diagonal" hermitian operator given by

$$
\begin{gathered}
{\left[B^{*}, B\right]\left(\ldots, x_{-1},\left[x_{0}\right], x_{1}, \ldots\right)=\left(\ldots, A_{-1}^{*} A_{-1}-A_{-2}, A_{-2}^{*}\right) x_{-1},} \\
\left.\left[\left(A_{0}^{*} A_{0}-A_{-1} A_{-1}^{*}\right) x_{0}\right],\left(A_{1}^{*} A_{1}-A_{0} A_{0}^{*}\right) x_{1}, \ldots\right) .
\end{gathered}
$$

Received by the editors June 25,1989 .

1980 Mathematics Subject Classification (1985 Revision). Primary 47A10, 47B20, 47B37.

Key words and phrases. Bilateral operator weighted shift, hyponormal operator, spectral picture, multiplicity, cyclic operator.

This research was partially supported by a grant of the National Science Foundation. 
It readily follows that $B$ is hyponormal (that is, $\left[B^{*}, B\right] \geq 0$ ) if and only if $A_{n}^{*} A_{n} \geq A_{n-1} A_{n-1}^{*}$ for all $n$.

Straightforward calculations show that $\|B\|=\sup _{n}\left\|A_{n}\right\|$ and that if $A_{n}$ admits a polar decomposition $A_{n}=U_{n} H_{n}$ with $U_{n}$ unitary $\left(0 \leq H_{n} \leq\left\|A_{n}\right\|\right)$, then $B$ is unitarily equivalent to the operator defined in the same way, with weights $\left\{H_{n}\right\}$. Unless otherwise stated, we shall assume that this is the case; that is, $0 \leq A_{n} \leq\|B\|$ for all $n$. In this case, the hyponormality of $B$ can be expressed as

$$
A_{n}^{2} \geq A_{n-1}^{2} \text { for all } n,
$$

and this implies that $\left\{A_{n}\right\}$ is a bounded, nondecreasing (see $[1,2,9]$ ) sequence of positive hermitian operators. Therefore, the strong limits

$$
\lim _{n \rightarrow \infty} A_{-n}=A_{-} \text {and } \lim _{n \rightarrow \infty} A_{n}=A_{+}
$$

are well defined [11, Problem 94]. Clearly, $0 \leq A_{-} \leq A_{n} \leq A_{+}$for all $n$; moreover, since $B$ is hyponormal, the spectral radius $\operatorname{sp}(B)$ of $B$ coincides with $\|B\|$, and either $A_{-}$is not invertible and $0 \in \sigma(B)$ or $A$ (and therefore $B)$ is invertible, $B^{-1}$ is a hyponormal and $\operatorname{sp}\left(B^{-1}\right)=\left\|A_{-}^{-1}\right\|[16$, Chapter 3].

Since $B$ is a bilateral operator weighted shift, every piece of $\sigma(B)$ is invariant under rotation [19]; therefore

$$
\sigma(B) \supset\left\{\lambda \in \mathbb{C}:|\lambda|=\|B\|=\left\|A_{+}\right\| \text {or }|\lambda|=\left\|A_{-}^{-1}\right\|^{-1}\right\} .
$$

( $\left\|A_{-}^{-1}\right\|^{-1}$ is defined equal to 0 in case $A_{-}$is not invertible).

In what follows, $\sigma_{\ell e}(T), \sigma_{\mathrm{re}}(T), \sigma_{e}(T)$ and $\sigma_{\ell \text { re }}(T)$ will denote the left essential spectrum of $T$, the right essential spectrum of $T$, the essential spectrum of $T$ and, respectively, $\sigma_{\ell e}(T) \cap \sigma_{\text {re }}(T)$. Recall that $\mathbb{C} \backslash \sigma_{\ell \text { re }}(T)$ coincides with $\rho_{\mathrm{s}-\mathrm{F}}(T)=\lambda \in \mathbb{C}: \lambda-T$ is not a semi-Fredholm operator $\}$. (The reader is referred to [7] for a definition and properties of the semi-Fredholm operators.)

Two operators, $A$ and $B$, acting on (not necessarily the same) Hilbert spaces are called approximately unitarily equivalent if $\left\|A-U_{k} B U_{k}^{*}\right\| \rightarrow 0 \quad(k \rightarrow \infty)$ for a suitable sequence of unitary mappings $\left\{U_{k}\right\}$ from the space of $B$ onto the space of $A$. (In symbols: $A \simeq_{a} B$. In this case the $U_{k}$ 's can be chosen so that, in addition, $A-U_{k} B U_{k}^{*}$ is a compact operator for all $k=1,2, \ldots$ [20].)

The reducing essential spectrum of an operator $T$ is the (possibly empty) compact set

$$
\sigma_{\text {red }}(T)=\left\{\lambda \in \mathbb{C}: T \simeq_{a} T \oplus \lambda\right\}
$$

$\left(\sigma_{\text {red }}(T) \subset \sigma_{\ell \text { re }}(T)\right.$; this notion was introduced by N. Salinas in [18]).

Let $U$ denote the bilateral shift on $\ell^{2}$; then

$$
U \simeq\left(\begin{array}{ll}
S & Z \\
0 & S^{*}
\end{array}\right)_{\ell_{-}^{2}}^{\ell_{+}^{2}}
$$

where $S$ is the unilateral shift on $\ell_{+}^{2}$.

With this notation in mind, we have the following. 
Theorem 1. Let $B$ be a bilateral operator weighted shift on $\ell^{2}(\mathscr{H})$ whose weight sequence $\left\{A_{n}\right\}\left(0 \leq A_{n} \leq\|B\|\right)$ admits strong limits in both directions:

$$
\lim _{n \rightarrow \infty} A_{-n}=A_{-} \text {and } \lim _{n \rightarrow \infty} A_{n}=A_{+} .
$$

Then $B$ is approximately unitarily equivalent to the direct sum of $B$ and the normal operator $U \otimes\left(A_{-} \oplus A_{+}\right)$. In particular,

$$
\sigma_{\text {red }}(B) \supset \sigma\left(U \otimes\left(A_{-} \oplus A_{+}\right)\right)=\left\{\lambda \in \mathbb{C}:|\lambda| \in \sigma\left(A_{-}\right) \cup \sigma\left(A_{+}\right)\right\} .
$$

Furthermore, if $1 \leq \operatorname{dim} \mathscr{H}<\infty$, then

$$
\sigma_{e}(B)=\sigma_{\ell \mathrm{re}}(B)=\sigma_{\mathrm{red}}(B)=\sigma\left(U \otimes\left(A_{-} \oplus A_{+}\right)\right) .
$$

Theorem 2. (i) Let $B$ be a hyponormal bilateral operator weighted shift, and let $r_{-}=\min \left\{r \in \sigma\left(A_{-}\right)\right\}$and $r_{+}=\max \left\{r \in \sigma\left(A_{+}\right)\right\} ;$then

$$
\sigma\left(U \otimes\left(A_{-} \oplus A_{+}\right)\right) \subset \sigma(B) \subset\left\{\lambda \in \mathbb{C}: r^{-} \leq|\lambda| \leq r^{+}\right\}
$$

and $B-\lambda$ is a semi-Fredholm operator with trivial kernel and negative index for all $\lambda \in \sigma(B) \cap \rho_{\mathrm{s}-\mathrm{F}}(B)$. Furthermore, if this set is not empty, and

$$
\alpha=\sup \left\{|\operatorname{ind}(\lambda-B)|: \lambda \in \sigma(B) \cap \rho_{\mathrm{s}-\mathrm{F}}(B)\right\}
$$

$(1 \leq \alpha \leq \infty)$, then the multiplicity of $B$ cannot be smaller than $\alpha+1$; that is, if $\ell^{2}(\mathscr{H})=\vee\left\{B^{k} x: k \geq 0, x \in \Gamma\right\}$ for some subset $\Gamma$ of $\ell^{2}(\mathscr{H})$, then $\operatorname{card}(\Gamma) \geq \alpha+1$.

(ii) If $B$ is a hyponormal operator of finite multiplicity, then the self-commutator, $\left[B^{*}, B\right]:=B^{*} B-B B^{*}$, of $B$ is a trace class operator and $\sigma_{e}(B)=$ $\sigma_{\ell \text { re }}(B)=\sigma_{\text {red }}(B)=\sigma\left(U \otimes\left(A_{-} \oplus A_{+}\right)\right)$.

Corollary 3. If $B$ is hyponormal and $1 \leq \operatorname{dim} \mathscr{H}=m<\infty$, then $\sigma(B)$ is the union of $m$ annuli of the form

$$
\Delta_{j}=\left\{\lambda \in \mathbb{C}: r_{j}^{-} \leq|\lambda| \leq r_{j}^{+}\right\}
$$

$\left(0 \leq r_{1}^{-} \leq r_{2}^{-} \leq \cdots \leq r_{m}^{-}, 0 \leq r_{1}^{+} \leq r_{2}^{+} \leq \cdots \leq r_{m}^{+}\right.$, and $r_{j}^{-} \leq r_{j}^{+}$for all $j=1,2, \ldots, m)$.

Either $B \simeq \sum \oplus_{j=1}^{m} r_{j}^{+} U$ is normal (all the annuli degenerate to circles, or to a point, and $B$ is cyclic if and only if all the radii $r_{j}^{+}$are positive and distinct), or at least one of the annuli has nonempty interior and $B$ is not cyclic. In the second case, $\sigma_{e}(B)=\sigma_{\ell \text { re }}(B)=\sigma_{\text {red }}(B)=\bigcup_{j=1}^{m} \partial \Delta_{j} ;$ moreover, for $\lambda \in \sigma(B) \backslash \sigma_{e}(B)$,

$$
-m \leq \operatorname{ind}(\lambda-B)=-\operatorname{card}\left\{j: \lambda \in \Delta_{j}\right\} \leq-1 .
$$

If $\operatorname{ker} B \neq\{0\}$, then $B \simeq B \oplus 0$, where 0 acts on an infinite dimensional space, and therefore $B$ and $B^{*}$ have infinite multiplicity. If $B$ has trivial kernel, then multiplicity $(B) \leq m+1$. 
J. B. Conway has raised the following question $[8$, p. 166]:

Open Problem 8.24. If $B$ is a hyponormal bilateral operator weighted shift, can $B$ have a cyclic vector?

(In the context of Conway's book, it is tacitly assumed that $B$ is hyponormal, but not normal.)

Theorem 2 implies that if all the weights are hermitian, then Conway's problem has a negative answer in a large number of cases. Indeed, if there is cyclic hyponormal (but not normal) bilateral operator weighted shift with positive weights, then $A_{+}^{2}-A_{-}^{2}$ must be a trace class operator, $\sigma\left(A_{+}\right)=\sigma\left(A_{-}\right)$, and $B$ is a compact perturbation of $U \otimes A_{+}$(and several other restrictions; see Corollary 5 below).

However, if we allow nonhermitian weights, Conway's problem has an affirmative answer: if $\mathscr{H}$ is infinite dimensional, then there exists a cyclic, nonnormal, hyponormal bilateral operator weighted shift $B$ on $\ell^{2}(\mathscr{H})$.

The example will be given in $\S 3$, which deals with the case when some of the weights do not have a polar decomposition with a unitary factor.

\section{Proof of the main Results}

Suppose $r \in \sigma\left(A_{+}\right), \varepsilon>0$, and $x \in \mathscr{H}$ is a unit vector such that $\left\|\left(A_{+}-r\right) x\right\|$ $<\varepsilon$; then $\left\|\left(A_{n}-r\right) x\right\|<2 \varepsilon$ for all $n \geq n_{0}(\varepsilon)$. Therefore, if $\hat{x}(n)=$ $\frac{1}{n}(\ldots, 0,[0], 0, \ldots, 0, x, x, \ldots, x, 0, \ldots)$ (the $x$ 's are located in coordinates $n^{2}+1$ through $\left.2 n^{2}\right)$, then $\hat{x}(n) \in \ell^{2}(\mathscr{H})$ is a unit vector, and a simple calculation shows that

$$
\begin{aligned}
& \|(B-r) \hat{x}(n)\|+\left\|(B-r)^{*} \hat{x}(n)\right\| \\
& \quad \leq \frac{2}{n}(\|B\|+r)+\frac{1}{n}\left(\sum_{k=n^{2}}^{2 n^{2}}\left\|\left(A_{n}-r\right) x\right\|^{2}\right)^{1 / 2}<3 \varepsilon,
\end{aligned}
$$

provided $n \geq n_{1} \geq n_{0}$.

Since $\varepsilon>0$ can be chosen arbitrarily small, we infer that $r$ is a reducing essential eigenvalue of $B$, in the sense of Salinas, and therefore $B \simeq_{a} B \oplus r l$.

Obviously, the same argument shows that $B \simeq_{a} B \oplus s l$ for each $s \in \sigma\left(A_{-}\right)$. Since $B \simeq e^{i \theta} B(0 \leq \theta \leq 2 \pi)$, it follows that $B \simeq_{a} B \oplus N$ for some normal operator $N$ with $\sigma(N)=\sigma_{e}(N)=\left\{\lambda \in \mathbb{C}:|\lambda| \in \sigma\left(A_{-}\right) \cup \sigma\left(A_{+}\right)\right\}$. Since this last set coincides with $\sigma\left(U \otimes\left[A_{-} \oplus A_{+}\right]\right)$, we deduce that $N \simeq_{a} U \otimes\left(A_{-} \oplus A_{+}\right)$, and therefore

$$
B \simeq_{a} B \oplus\left[U \otimes\left(A_{-} \oplus A_{+}\right)\right]
$$

It readily follows that $\sigma_{\text {red }}(B)$ includes the spectrum of $U \otimes\left(A_{-} \oplus A_{+}\right)$.

This proves the first statement.

Now assume that $\mathscr{H}$ is finite dimensional. Observe that

$$
B=\left(\begin{array}{cc}
B_{+} & F \\
0 & B_{-}
\end{array}\right)_{\ell_{-}^{2}(\mathscr{H})}^{\ell_{+}^{2}(\mathscr{H})},
$$


where

$$
F=\left(\begin{array}{cc}
0 & 0 \\
A_{1} & 0
\end{array}\right)
$$

(with respect to suitable decompositions of $\ell_{+}^{2}(\mathscr{H})$ and $\ell_{-}^{2}(\mathscr{H})$ as direct sums of one copy of $\mathscr{H}$ and its orthogonal complement), so that rank $F \leq m$, and $B_{+}$and $B_{-}$are unitarily equivalent to compact perturbations of $S \otimes A_{+}$, and, respectively, $S^{*} \otimes A_{-}$(because, in this case, $\left\|A_{-n}-A_{-}\right\| \rightarrow 0$ and $\left\|A_{n}-A_{+}\right\| \rightarrow 0$ as $n \rightarrow \infty$ !).

Hence,

$$
B \simeq\left(S \otimes A_{+}\right) \oplus\left(S^{*} \otimes A_{-}\right)+K
$$

where $K \in \mathscr{K}$ (= the ideal of all compact operators).

Since $\pi\left[\left(S \otimes A_{+}\right) \oplus\left(S^{*} \otimes A_{-}\right)\right]$is a normal element of the quotient Calkin algebra $\mathscr{L}\left(\ell^{2}(\mathscr{H})\right) / \mathscr{K}$ (see [7]), we conclude that

$$
\begin{aligned}
\sigma_{e}(B) & =\sigma_{\ell \mathrm{re}}(B)=\sigma_{e}\left(S \otimes A_{+}\right) \cup \sigma_{e}\left(S^{*} \otimes A_{-}\right) \\
& =\left\{\lambda \in \mathbb{C}:|\lambda| \in \sigma\left(A_{-}\right) \cup \sigma\left(A_{+}\right)\right\} .
\end{aligned}
$$

The proof of Theorem 1 is now complete.

The author conjectures that the conditions " $A_{-n} \rightarrow A_{-}$and $A_{n} \rightarrow A_{+}$ strongly" can be relaxed to " $A_{-n} \rightarrow A_{-}$and $A_{n} \rightarrow A_{+}$weakly".

In general, the inclusion $\sigma_{\ell \text { re }}(B) \supset\left\{\lambda \in \mathbb{C}:|\lambda| \in \sigma\left(A_{-}\right) \cup \sigma\left(A_{+}\right)\right\}$is proper, even if $B$ is hyponormal. For instance, if $\mathscr{H}=L^{2}([0, \infty)), A_{n}=1$ for $-\infty<n<3$, and $A_{n}=1+2 P_{L^{2}([0, n))}+P_{L^{2}([n, 3 n))}(n \geq 3)$, and $\hat{x}(n)=$ $\frac{1}{n}\left(\ldots, 0,[0], 0, \ldots, 0, e_{n}, e_{n}, \ldots, e_{n}, 0, \ldots\right)\left(e_{n}\right.$ is the characteristic function of the interval $\left[2 n^{3}, 2 n^{3}+1\right)$ and the $k$ th coordinate is equal to $e_{n}$ for $n^{3}+1 \leq k \leq n^{3}+n^{2}$, and zero otherwise), then $B$ is hyponormal and invertible,

$$
A_{-n} \rightarrow 1 \text { and } A_{n} \rightarrow 3 \text { (strongly, as } n \rightarrow \infty \text { ), }
$$

but

$$
\left\|(B-2)^{*} \hat{x}(n)\right\| \leq\|(B-2) \hat{x}(n)\|<\frac{2}{n} \rightarrow 0 \quad(n \rightarrow \infty),
$$

whence we deduce that $B \simeq_{a} B \oplus 2 U$, although $2 \notin \sigma\left(A_{-}\right) \cup \sigma\left(A_{+}\right)=\{1,3\}$.

If $\left\|A_{-n}-A_{-}\right\|+\left\|A_{n}-A_{+}\right\| \rightarrow 0 \quad(n \rightarrow \infty)$, then we can prove that $\sigma_{\text {red }}(B) \supset$ $\sigma\left(U \otimes\left(A_{-} \oplus A_{+}\right)\right)$by a more direct argument, by using Berg's technique [3], as described in [14, Chapter 2].

Conjecture 4. If $\left\|A_{-n}-A_{-}\right\|+\left\|A_{n}-A_{+}\right\| \rightarrow 0 \quad(n \rightarrow \infty)$, then $\sigma_{\ell \mathrm{re}}(B)=$ $\sigma_{\text {red }}(B)=\sigma\left(U \otimes\left(A_{-} \oplus A_{+}\right)\right)$.

Proof of Theorem 2. (i) The first inclusion follows from Theorem 1, and the second one follows from the fact that $\|B\|=\operatorname{sp}(B)$ for all hyponormal operators (see Introduction; [16]).

If $B$ is hyponormal and $\mu$ is an eigenvalue of $B$, then $B \simeq B_{\mu} \oplus \mu l_{\mathbb{C}}$ [16]. Since $B \simeq e^{i \theta} B$, the same argument applies to every point of the circle of radius 
$|\mu|$. The separability of $\mathscr{H}$ makes this impossible, unless $\mu=0$. In this case, $\operatorname{ker} A_{p} \neq\{0\}$ for some $p$. Whence it readily follows that $\operatorname{ker} A_{p} \subset \operatorname{ker} A_{n} \neq\{0\}$ for all $n \geq p$; and therefore $B \simeq B \oplus 0$, where 0 acts on an infinite dimensional space (and therefore $B$ and $B^{*}$ have infinite multiplicity; clearly, neither $B$ nor $B^{*}$ can be a semi-Fredholm operator).

Thus, if $\lambda \in \sigma(B) \cap \rho_{\mathrm{s}-\mathrm{F}}(B)$, then $\operatorname{ker}(\lambda-B)=\{0\}$ and $-\infty \leq-h=$ $\operatorname{ind}(\lambda-B) \leq-1$. But $B$ cannot be a semi-Fredholm operator of negative index. Thus, if $\Omega$ is a component of

$$
\left\{\lambda \in \rho_{\mathrm{s}-\mathrm{F}}(B): \operatorname{ind}(\lambda-B)=-h\right\},
$$

then $\Omega$ is either an annulus or a punctured disk. In either case, $\Omega$ is not simply connected, and therefore (using [13, Theorem 1] or [15])

$$
\text { multiplicity }(B) \geq h+1 \text {, }
$$

whence we obtain the last statement, by taking supremum over all possible $\lambda$ 's.

(ii) If $B$ is hyponormal and has finite multiplicity, the Berger-Shaw trace inequality implies that $\left[B^{*}, B\right]$ is a trace class operator; this is equivalent to saying that

$$
A_{+}^{2}-A_{-}^{2} \text { is trace class }
$$

(trace norm $\left(A_{+}^{2}-A_{-}^{2}\right)=\operatorname{trace}\left[B^{*}, B\right] \leq \frac{1}{\pi}$ area $\sigma(B) \times$ multiplicity $(B)$; see $[4$, 5]), and therefore compact. In particular, $\sigma_{e}\left(A_{+}^{2}\right)=\sigma_{e}\left(A_{-}^{2}\right)$, whence we obtain $\sigma_{e}\left(A_{+}\right)=\sigma_{e}\left(A_{-}\right)$. (The Berger-Shaw trace inequality applies to hyponormal operators of finite rational multiplicity. But the difference is irrelevant here, because rat. multiplicity $(T) \leq \operatorname{multiplicity}(T) \leq \operatorname{rat}$ multiplicity $(T)+1$ for every operator $T$ [13, Proposition 2].)

If $A_{-}$is bounded below by $\delta>0$ and $\gamma$ is the (positively oriented) circle of radius $\|B\|^{2}-(\delta / 2)^{2}$ centered at $\|B\|^{2}$, then by using the second resolvent equation [11], [17], we see that

$$
\begin{aligned}
A_{+}-A_{-} & =\frac{1}{2 \pi i} \int_{\gamma} \sqrt{\lambda}\left\{\left(\lambda-A_{+}^{2}\right)^{-1}-\left(\lambda-A_{-}^{2}\right)^{-1}\right\} d \lambda \\
& =\frac{1}{2 \pi i} \int_{\gamma} \sqrt{\lambda}\left(\lambda-A_{+}^{2}\right)^{-1}\left(A_{+}^{2}-A_{-}^{2}\right)\left(\lambda-A_{-}^{2}\right)^{-1} d \lambda
\end{aligned}
$$

is also a trace class operator.

If $A_{-}$is not bounded below, then

$$
\left\|A_{-}-\left(A_{-}^{2}+\varepsilon l\right)^{1 / 2}\right\| \rightarrow 0,\left\|A_{+}-\left(A_{+}^{2}+\varepsilon l\right)^{1 / 2}\right\| \rightarrow 0(\varepsilon \rightarrow 0),
$$

and

$$
\left.\| A_{+}-A_{-}\right)-\left[\left(A_{+}^{2}+\varepsilon l\right)^{1 / 2}-\left(A_{-}^{2}+\varepsilon l\right)^{1 / 2}\right] \| \rightarrow 0(\varepsilon \rightarrow 0),
$$

whence we infer that $A_{+}-A_{-}$is a positive hermitian compact operator. (It is not difficult to check that we also have $\left\|A_{-n}-A_{-}\right\|+\left\|A_{n}-A_{+}\right\| \rightarrow 0$, as $n \rightarrow \infty$.) 
Since $\left[\left(B \oplus B^{*}\right)^{*}, B \oplus B^{*}\right]=\left[B^{*}, B\right] \oplus\left[B, B^{*}\right]$ is compact and (by using the invariance under rotation of every part of the spectrum of $\sigma(B))$ ind $\left(\lambda-B \oplus B^{*}\right)$ $=0$ for all $\lambda \in \rho_{\mathrm{s}-\mathrm{F}}(B)=\rho_{\mathrm{s}-\mathrm{F}}\left(B^{*}\right)$, the Brown-Douglas-Fillmore theorem implies that $B \oplus B^{*}$ is a compact perturbation of a normal operator $M$, and $\sigma_{e}(B)=\sigma_{\text {red }}(B)=\sigma_{e}\left(B^{*}\right)=\sigma_{\text {red }}\left(B^{*}\right)=\sigma(M)=\sigma_{e}(M)[6]$.

Observe that a suitable compact perturbation of $B$ can be written as an operator matrix $\left(C_{i j}\right) \quad(-\infty<i, j<\infty)$ with respect to a decomposition $\ell^{2}(\mathscr{H})=\sum \oplus\left\{\mathscr{H}_{j}:-\infty<j<\infty ; \mathscr{H}_{j} \simeq \mathscr{H}\right.$ for all $\left.j\right\}$, where $C_{i, i+1}=A_{+}$, or $A_{-}$, according to whether $i \geq 0$ or $i<0$, and $C_{i j}=0$ if $j \neq i+1$. Similarly, $B^{*}$ can be written as an operator matrix of exactly the same form, with $C_{i, i+1}=A_{-}$, or $A_{+}$, according to whether $i \geq 0$, or $i<0$. Given $\varepsilon>0$, we can use Berg's technique [3;14, Chapter 2] in order to approximate $B \oplus B^{*}$ in less than $\varepsilon$ by an operator $T \simeq R \oplus V$, where $R \simeq U \otimes A_{-}$and $V$ is a bilateral operator weighted shift such that all but finitely many weights are equal to $A_{+}$and all the weights satisfy $A_{-} \leq V_{n} \leq A_{+}$. Clearly, $T$ is unitarily equivalent to a compact perturbation of $U \otimes\left(A_{-} \oplus A_{+}\right)$, and therefore $\sigma_{e}(T)=\sigma_{\text {red }}(T)=\sigma_{e}\left(U \otimes\left(A_{-} \oplus A_{+}\right)\right)$.

Since $B \oplus B^{*}$ and its approximation $T$ project into normal elements of the Calkin algebra, and since $a \rightarrow \sigma(a)$ is always a continuous mapping, when restricted to normal elements of any $C^{*}$-algebra, we conclude that $\sigma_{e}(B)=$ $\sigma_{e}\left(B \oplus B^{*}\right)=\sigma_{e}\left(U \otimes\left(A_{-} \oplus A_{+}\right)\right)=\sigma\left(U \otimes\left(A_{-} \oplus A_{+}\right)\right)$.

Let $B$ be as in Theorem 2 (ii), so that $\sigma_{e}(B)$ is the union of $\sigma\left(U \otimes A_{-}\right)$ and $\sigma\left(U \otimes A_{+}\right)$. Recall that $A_{+}-A_{-}$is compact, so that $\sigma\left(A_{+}\right)$and $\sigma\left(A_{-}\right)$ have exactly the same nonisolated points. Assume that $r \in \sigma\left(A_{-}\right) \backslash \sigma\left(A_{+}\right)$; then $r$ is an isolated point of $\sigma\left(A_{-}\right)$, and therefore the circle of radius $r$ is a clopen subset of $\sigma_{e}(B)$. If this circle is also a clopen subset of $\sigma(B)$, then the hyponormal operator $B$ is unitarily equivalent to $r U \oplus B^{\prime}$, for some hyponormal operator $B^{\prime}$ with $\sigma\left(B^{\prime}\right)=\sigma(B) \backslash \sigma(r U)$ [16]. But this would imply that $r \in \sigma\left(A_{+}\right)$, a contradiction.

Hence, the circle of radius $r$ cannot be a clopen subset of $\sigma(B)$. It readily follows that if $\varepsilon>0$ is small enough, then either $(r-\varepsilon)-B$, or $(r+\varepsilon)-B$ is a semi-Fredholm operator of negative index, whence we infer that $B$ cannot be cyclic.

The same argument shows that $B$ cannot by cyclic if $\sigma\left(A_{+}\right)$is not included in $\sigma\left(A_{-}\right)$.

Furthermore, (even if $\sigma(B)=\sigma_{e}(B)$ ) if $B^{*}$ has a nonzero eigenvalue, by proceeding along the lines of [12], we can still conclude that $B$ cannot be a cyclic operator.

Hence, we have obtained the following.

Corollary 5. If $B$ is a cyclic, hyponormal, bilateral operator weighted shift with positive weights $\left\{A_{n}\right\}$, then

(i) $B^{*}$ has no eigenvalues, 
(ii) $A_{+}^{2}-A_{-}^{2}$ is a positive hermitian operator whose trace norm does not exceed $\frac{1}{\pi}$ area $\sigma(B)$,

(iii) $\sigma\left(A_{+}\right)=\sigma\left(A_{-}\right)$and $\sigma(B)=\sigma_{e}(B)=\sigma_{\ell \text { re }}(B)=\sigma_{\text {red }}(B)=\sigma\left(U \otimes A_{+}\right)=$ $\sigma\left(U \otimes A_{-}\right)$, and

(iv) $\operatorname{ker}\left(r-A_{-}\right)=\operatorname{ker}\left(r-A_{+}\right)$and this subspace has dimension one, for each isolated point $r$ of $\sigma\left(A_{+}\right)$.

The last statement follows from the fact that $U \oplus U$ is not cyclic. The author strongly suspects that if $B$ is cyclic, then it is necessarily a normal operator. (In this case, $B$ has the form $B=U \otimes A$ for some cyclic positive hermitian operator $A$ with trivial kernel.)

Proof of Corollary 3. The spectral structure, and most of the other statements, follow immediately from Theorems 1 and 2 (ii).

The only point not contained in the theorems is multiplicity. Assume that $\operatorname{ker} B$ is trivial; then all the weights are invertible and $B_{-}$has a cyclic vector $x_{-} \in \ell^{2}(\mathscr{H})$. (This follows from the same argument that shows that $\left(S^{*} \otimes 1\right)$ has a cyclic vector; see, e.g., [11].) Now it is straightforward to check that if $\left\{e_{1}, e_{2}, \ldots, e_{m}\right\}$ is a basis of $\mathscr{H}$ and $\hat{y}_{j}=\left(\ldots, 0,0,\left[e_{j}\right], 0,0, \ldots\right)(j=$ $1,2, \ldots, m)$, then

$$
\ell^{2}(\mathscr{H})=\vee\left\{B^{k}\left(x_{-} \oplus 0\right), B^{k} y_{j}: k \geq 0,1 \leq j \leq m\right\} .
$$

Therefore, multiplicity $(B) \leq m+1$.

\section{The CASE WhEN THE WEIGHTS DO NOT haVe A GOOD FACTORIZATION}

Throughout this section we shall assume that $\mathscr{H}$ is infinite dimensional, $B$ is hyponormal, and some of the weights (say, $A_{0}$ ) cannot be factored as Unitary $\times$ Positive; then either $\operatorname{ker} A_{0}$ is nontrivial, or $\operatorname{ker} A_{0}^{*}$ is nontrivial, $A_{-1} A_{-1}^{*} \leq A_{0}^{*} A_{0}$ and $A_{0} A_{0}^{*} \leq A_{1}^{*} A_{1}$.

If $\operatorname{ker} A_{0} \neq\{0\}$, then $\operatorname{ker} B^{*} \supset \operatorname{ker} B \neq\{0\}$ and $B \simeq B^{\prime} \oplus 0_{\operatorname{ker} B}$. If $\operatorname{ker} B$ is infinite dimensional, then $B$ and $B^{*}$ have infinite multiplicity; if $B$ is cyclic, then $\operatorname{ker} B$ must be, at most, one dimensional, and this implies that $\operatorname{ker} A_{n}=$ $\{0\}$ for all $n \neq 0$. For example, if $A_{0}=S^{*}, A_{-1}=S$, and $A_{n}=1$ for all $n \neq 0,-1$, then $B \simeq(U \otimes 1) \oplus 0_{\mathbb{C}}$ (normal, not cyclic). If $H$ is positive hermitian, cyclic, and ker $H$ is trivial, and we choose $A_{0}=H S^{*}, A_{-1}=S H$, and $A_{n}=H$ for all $n \neq 0,-1$ then

$$
B \simeq(U \otimes H) \oplus 0_{\mathbb{C}} \text { (normal and cyclic) } .
$$

Example 6. Define $B$ as usual, with $A_{-n}=J(n>0), A_{0}=S J$ and

$$
A_{n}=\left(\begin{array}{ll}
1 & 0 \\
0 & J
\end{array}\right)_{\left(\operatorname{ker} S^{*}\right)^{\perp}}^{\left(\operatorname{ker} S^{*}\right)} \quad(n>0),
$$

where $J$ is the diagonal hermitian operator with (simple) eigenvalues $2,3 / 2$, $4 / 3,5 / 4, \ldots$. Then $B$ is a cyclic, nonnormal, hyponormal bilateral operator weighted shift; moreover, $B \simeq\left\{\sum \bigoplus_{n=1}^{\infty}\left(1+\frac{1}{n}\right) U\right\} \oplus S$. 
Proof. Write $B$ as an upper triangular matrix with respect to the decomposition $\ell^{2}(\mathscr{H}) \simeq \sum \bigoplus\left\{\mathscr{H}_{k}:-\infty<k<\infty\right\}$. A straightforward analysis shows that

$$
B \simeq\left\{\sum \bigoplus_{n=1}^{\infty}\left(1+\frac{1}{n}\right) U\right\} \oplus S
$$

which is obviously hyponormal, but not normal.

It only remains to show that $B$ is cyclic. Think of $U$ as the "multiplication by $e^{i \theta}$ " on $L^{2}(\partial \mathbb{D}, d m)$ ( $d m=$ normalized linear Lebesgue measure on the unit circle), and $S$ is the unilateral shift with respect to the orthonormal basis $\left\{e_{j}=\lambda^{j}\right\}_{j=0}^{\infty}$ of $H^{2}(\partial \mathbb{D})$; then $B$ is the operator "multiplication by $\lambda$ " on

$$
\left\{\sum \bigoplus_{n=1}^{\infty} L^{2}\left(\Delta_{n}, d m_{n}\right)\right\} \oplus H^{2}(\partial \mathbb{D})
$$

where $\Delta_{n}$ is the circle of radius $1+\frac{1}{n}$ and $d m_{n}$ is the normalized linear Lebesgue measure on this circle $(n=1,2, \ldots)$. Let $\Delta_{n}^{+}, \Delta_{n}^{-}$denote the intersection of $\Delta_{n}$ with the upper half-plane and, respectively, with the lower halfplane. By Runge's theorem [10], there exist sequences of polynomials $\left\{p_{n k}\right\}_{k=1}^{\infty}$ $(n=1,2, \ldots)$ such that

$$
\left|p_{n k}(\lambda)-1\right|<1 / k \text { for all } \lambda \in \Delta_{n}^{+}
$$

and

$$
\left|p_{n k}(\lambda)\right|<1 / k \text { for } \lambda \in \Delta_{n+1} \cup\left\{\zeta \in \Delta_{n}^{-}:-\pi+\frac{1}{k}<\arg \zeta<-\frac{1}{k}\right\}
$$

(so that $p_{n k}(\lambda) \rightarrow 0$, as $k \rightarrow \infty$, uniformly on the closed disk of radius $1+$ $1 /(n+1), n=1,2, \ldots)$.

Now we use an argument very close to [13, Example 5, pp. 75-76]. Let $f_{n} \in L^{2}\left(\Delta_{n}, d m_{n}\right)$ be a function such that $f_{n} \equiv 1$ on $\Delta_{n}^{+}, 0<f(\lambda) \leq 1, f$ is continuous on $\Delta_{n}^{-}$and approaches to 0 when $\lambda$ approaches the real axis from below, and

$$
\int_{\Delta_{n}}\left|g_{n}(\lambda)-p_{n k}(\lambda) f_{n}(\lambda)\right|^{2} d m_{n}<2 / k^{2}(k=1,2, \ldots),
$$

where $g_{n}$ denotes the characteristic function of $\Delta_{n}^{+}(n=1,2, \ldots)$.

Finally, define

$$
y=\left\{\sum \bigoplus_{n=1}^{\infty} 2^{-n} f_{n}\right\} \oplus e_{0} .
$$

Let $\mathscr{M}$ be the closed linear span of $\left\{B^{k} y\right\}_{k=0}^{\infty}$. Clearly,

$$
g_{1}=(\text { norm }) \lim _{k \rightarrow \infty} 2 p_{l k}(B) y \in \mathscr{M},
$$

whence we deduce that $L^{2}\left(\Delta_{1}^{+}, d m_{1}\right) \oplus\{0\}=\bigvee\left\{B^{k} g_{1}\right\}_{k=0}^{\infty} \subset \mathscr{M}$; moreover, since $f_{1}-g_{1}$ is a continuous function, strictly positive on $\Delta_{n}^{-}$, a new application 
of Runge's theorem shows that $f_{1}-g_{1} \in \mathscr{M}$, and therefore

$$
L^{2}\left(\Delta_{1}, d m_{1}\right) \oplus\{0\}=L^{2}\left(\Delta_{1}^{+}, d m_{1}\right) \oplus L^{2}\left(\Delta_{1}^{-}, d m_{1}\right) \oplus\{0\} \subset \mathscr{M} .
$$

A formal repetition of the same argument (with $y$ replaced by $y_{1}=y-$ $\left.2^{-1} f_{1} \oplus 0\right)$ indicates that $L^{2}\left(\Delta_{2}, d m_{2}\right) \oplus\{0\}$ is also included in $\mathscr{M}$, and therefore

$$
L^{2}\left(\Delta_{1}, d m_{1}\right) \oplus L^{2}\left(\Delta_{2}, d m_{2}\right) \oplus\{0\} \subset \mathscr{M} .
$$
fore,

By induction, we deduce that $\left\{\sum \bigoplus_{n=1}^{\infty} L^{2}\left(\Delta_{n}, d m_{n}\right)\right\} \oplus\{0\} \subset \mathscr{M}$. There-

$$
0 \oplus e_{0}=y-\left\{\sum \bigoplus_{n=1}^{\infty} 2^{-n} f_{n}\right\} \oplus 0 \in \mathscr{M},
$$

whence we conclude that $\{0\} \oplus H^{2}(\partial \mathbb{D}) \subset \mathscr{M}$.

Hence, $y$ is a cyclic vector for $B$.

\section{REFERENCES}

1. T. Ando, Topics of operator inequalities, mimeographed notes, Hokkaido Univ., Sapporo, Japan, 1978.

2. __, On some operator inequalities, Math. Ann. 279 (1987), 157-159.

3. I. D. Berg, On approximation of normal operators by weighted shifts, Michigan Math. J. 21 (1974), 377-383.

4. C. A. Berger and B. I. Shaw, Self-commutators of multicyclic hyponormal operators are always trace class, Bull. Amer. Math. Soc. 79 (1973), 1193-1199.

5. __ Intertwining, analytic structure, and the trace norm estimate, Proceedings of a conference on operator theory, Halifax, Nova Scotia, 1973; Lecture Notes in Math., vol. 345, Springer-Verlag, Berlin, Heidelberg, and New York, 1973, pp. 1-6.

6. L. G. Brown, R. G. Douglas, and P. A. Fillmore, Unitary equivalence modulo the compact operators and extensions of $C^{*}$-algebras, Proceedings of a conference on operator theory, Halifax, Nova Scotia, 1973; Lecture Notes in Math., vol. 345, Springer-Verlag, Berlin, Heidelberg and New York, 1973, pp. 58-128.

7. S. R. Caradus, W. E. Pfaffenberger, and B. Yood, Calkin algebras and algebras of operators on Banach spaces, Lecture Notes in Pure and Appl. Math., vol. 9, Dekker, New York, 1974.

8. J. B. Conway, Subnormal operators, Res. Notes in Math., vol. 51, Pitman, Boston, London, and Melbourne, 1981.

9. W. Donoghue, Monotone matrix functions and analytic continuation, Springer-Verlag, Berlin, Heidelberg, and New York, 1974.

10. T. W. Gamelin, Uniform algebras, Prentice-Hall, Englewood Cliffs, NJ, 1969.

11. P. R. Halmos, A Hilbert space problem book, Van Nostrand, Princeton, NJ, 1967.

12. D. A. Herrero, Eigenvectors and cyclic vectors for bilateral weighted shifts, Rev. Un. Mat. Argentina 26 (1972), 24-41.

13. _ On multicyclic operators, Integral Equations Operator Theory 1 (1978), 57-102.

14. _ Approximation of Hilbert space operators I, Res. Notes in Math., vol. 72, Pitman, Boston, London, and Melbourne, 1982.

15. _ The Fredholm structure of an n-multicyclic operator, Indiana Univ. Math. J. 36 (1987), 549-566.

16. C. R. Putnam, Commutation properties of Hilbert space operators and related topics, Ergeb. Math. Grenzgeb. (3) 36, Springer-Verlag, New York, 1967. 
17. F. Riesz and B. Sz.-Nagy, Functional analysis, Ungar, New York, 1955.

18. N. Salinas, Reducing essential eigenvalues, Duke Math. J. 40 (1973), 561-580.

19. A. L. Shields, Weighted shift operators and analytic function theory, Math. Surveys Monogr. 13 (1974), 49-128.

20. D. Voiculescu, A non-commutative Weyl-von Neumann theorem, Rev. Roumaine Math. Pures Appl. 21 (1976), 97-113.

Department of Mathematics, Arizona State University, Tempe, Arizona 85287 